\title{
Risk factors for induction failure of standard chemotherapy with anthracycline and cytarabine in Acute Myeloid Leukemia Patients
}

\author{
Christian F Jehn $^{1}$, Marc Pannenbeckers ${ }^{1}$, Anna Klapproth ${ }^{1}$, Farouk Dahmash ${ }^{1}$, Hans Salwender ${ }^{1}$, Anju Singh ${ }^{1}$, Yana Shikova ${ }^{1}, J^{2 a r a}$ Pascale \\ Schlichting ${ }^{1}$, Silke Meyer ${ }^{1}$, Cornelius Niggemann ${ }^{2}$, Mathias Vierbuchen ${ }^{2}$ and Ahmet H Elmaagacli* ${ }^{1 *}$ \\ ${ }^{1}$ Department of Hematology/Oncology and Stemcelltransplantation, AK St. Georg, Hamburg \\ ${ }^{2}$ Department of Pathology, AK St. Georg, Hamburg, Germany
}

\begin{abstract}
Induction therapy (IT) with anthracycline and cytarabine (DA) is, despite a new era with targeted therapies such as FLT-3- or IDH-1/2- inhibitors, still the backbone of treatment for acute myeloid leukemia (AML). In this retrospective study we investigated possible risk factors for induction failure (IF) in 109 AML-patients, who were consecutively treated between 2013 and 2018 at our institution. We evaluated all patients at diagnosis for CMV IgG-status, LDH-value, platelet count, blast count in bone marrow (BM), Sorror comorbidity score (range $0-6$ ), age ( $>70$ years), cytogenetic risk factors according to the ELN classification (favourable [ $\mathrm{n}=15$ ], intermediate [ $\mathrm{n}=56$ ], or high risk [n=38]), occurrence of biclonal AML detected by flow cytometry and extramedullary AML-manifestation. In 43 (39\%) patients an IF was observed. 38 of these patients went on to received a salvage therapy with idarubicin and fludarabine $(\mathrm{n}=30)$ or directly to allotransplant $(\mathrm{n}=8)$, whereas 5 patients received only best supportive care. Only age $>70$-years $(p=0.020$, odds ratio $[O R] 2.5)$, cytogenetic adverse risk classification $(p=0.014$; OR 3.21$)$, Sorror comorbidity score of $>2$, $(p=0.019$, OR 2.72$)$, and $>40 \%$ blasts in BM ( $\mathrm{p}=0.004$; OR 3.64), had influence on the occurrence of IF after DA. Patients with IF or adverse cytogenetics and without subsequent transplant had a worse prognosis (2-year OS for IF $19.4 \%+16.8 \%$ versus $86.9 \%+7.6 \%, \mathrm{p}=0.0001)$. In multivariate analyses for OS only transplantation (HR 0.37; [95\% CI 0.19-0.76], p=0.007) and blasts >40\% in BM (HR 2.24, [95\% CI $1.07-4.68$ ], p= 0.032), but not IF were identified as independent risk factors.
\end{abstract}

\section{Introduction}

For more than 46 years the induction therapy with an anthracycline and cytarabine (DA) has remained worldwide the most frequently used regimen in patients with acute myeloid leukemia (AML) worldwide [1]. Although the regimen is effective in the induction of complete remission in AML in many of the cases, induction failure (IF) after DA still occurs frequently [1-3]. Some risk factors for IF have been identified already [2]. These include older age of patients and the detection of cytogenetic high-risk characteristics according to the European Leukemia Network (ELN) classification [3-6]. Generally, age over 60 years is considered to be a risk factor for IF and poorer outcome of AML [2]. Due to fact that the median age of onset of AML is over 70 years and significant improvements have been achieved in supportive care over the last decades, more and more elderly patients are treated in curative intention with intensive induction chemotherapy for AML and proceed to allogenic transplantation [7]. This could be a cause for an increased occurrence of IF after DA over the last years because advanced age is often accompanied by more adverse cytogenetics [3]. The identification of additional risk factors besides advanced age and unfavourable cytogenetics is of importance due to the fact that a reduced performance status and multiple comorbidities in older patients often prevent the application of additional courses of toxic salvage chemotherapies for refractory AML after DA was given. Consolidating allogenic SCT transplantation (alloSCT) has become an increasing option in this elderly patient population due to lower toxicity and therapy associated mortality of reduced conditioning regimes (RIC), thereby adding an effective component of AML therapy in this elderly population, the Graft vs. leukemia effect (GvL). With alloSCT as a realistic option for consolidation therapy in elderly AML patients, new approaches in up-front induction chemotherapy could be beneficiary. New approved drug combinations like Vyxeos or targeted therapy combinations with Venetoclax and Azacitidine have shown feasible tolerability and promising efficacy in elderly AML patients, with a remarkable increase in patients proceeding to transplant, compared to other traditional anthracycline-free induction regimes.

So, the aim of this retrospective single centre study was first, to investigate risk factors for IF of standard chemotherapy with DA in 109 patients with AML, who were consecutively treated between 2013 and 2018 at our institution. Secondly, to analyse the impact of these risk factors on outcome of our AML-patients.

\section{Patients and methods}

Patient's characteristics and eligibility. The study population encompasses 109 AML patients who were treated with one or two courses of induction therapies with 3 days of an anthracycline

${ }^{\star}$ Correspondence to: Ahmet H. Elmaagacli, M.D, Hematology/Oncology and Stem cell transplantation, Asklepios Klinik St. Georg, Lohmühlenstr. 5, 20099 Hamburg, Germany, Tel. +49-40-18181-852005; Fax: +49-40-1818852226; E-mail: ahmet.elmaagacli@uni-due.de

Key words: induction failure, acute myeloid leukemia, risk factors

Received: November 21, 2019; Accepted: December 03, 2019; Published: December 06, 2019 
(eg, daunorubicin $45-60 \mathrm{mg} / \mathrm{m} 2$ or an alternative anthracycline at equivalent dose), and 7 days of cytarabine (100-200 $\mathrm{mg} / \mathrm{m} 2$ continuous intravenously $[7+3 \mathrm{DA}])$. This patient cohort was selected from a total of 122 AML-patients (thus comprising $89 \%$ of all AML-patients), who received DA between January 2013 and December 2017 at our department. Thirteen patients were excluded from the analysis due to lack of data.

We evaluated in all our study-patients at time point of diagnosis CMV, IgG serostatus (positive versus negative), LDH-value (norm or upper norm), platelet count (over $50 \mathrm{n} / \mathrm{L}$ or below), blast count in bone marrow, ECOG 0-2 versus 3-4, Sorror comorbidity score (range $0-6$ ), age ( $<70$ years or above), cytogenetic risk factors according to the ELN classification (favourable $[n=15]$, intermediate $[n=56]$, or high risk $[n=38])$, occurrence of biclonal AML detected by flow cytometry and extramedullary manifestation of AML. Biclonal AML was defined as the detection of a further different AML clone covering at least $2 \%$ of all blasts at time point of diagnosis.

In all transplanted patients complete allele identity at the HLA loci A, B, C, DRB1 and DQB1 as confirmed by high-resolution genotyping in unrelated donor/recipient pairs and by low resolution genotyping with documented familial segregation of the 10 HLA antigens in sibling pairs were evaluated as previously published [8]. Patients were included in the study only by availability of the primary karyotype or on qualitative analysis of disease-specific molecular markers in case of normal karyotype AML for patients in complete remission (CR) according to the diagnostic standards in the respective time periods.

All patients gave their written informed consent to all aspects of induction chemotherapy with DA, if applicable to salvage chemotherapy and stem cell transplantation procedure in accordance to the institutional standards of our department, which are in accordance with the standards of Good Clinical Practice and the Declaration of Helsinki. The study protocols were approved by the Hamburger Ethics Committee (Ärztekammer Hamburg, WF-061/19). No experimental drugs or therapy were used. Patient, donor, disease and transplant characteristics are summarized in Table 1.

\section{Definition of IF and haematological relapse after transplant}

IF was defined as non-responsiveness of one induction therapy with $>30 \%$ blasts regardless of cellularity or non-achievement of complete remission after two induction therapies. Standard marrow morphology and hematologic criteria were used for the evaluation of the pre- and post-transplant disease stage, post-transplant treatment response, and leukemic relapse after transplantation. The prognostic categorization of the genetic disease profile relied on the cytogenetic and molecular genetic detection methods representing the diagnostic standards in the respective time periods of this study and was retrospectively performed according to the recommendations of the European Leukemia Network expert panel [9]. Hematologic leukemic relapse was diagnosed in patients, who were in CR before or attained CR after alloSCT. During the first year after alloSCT marrow examinations were routinely performed at days 28 and 100 , and at 12 months $[8,10,11]$.

\section{Transplant}

From the start of the induction chemotherapy until discharge, all patients were treated in reverse isolation single rooms equipped with high-efficacy particular air (HEPA) filtration. Additional measures for infection prevention and for supportive therapy complied

Table 1. Patient's characteristics

\begin{tabular}{|c|c|c|c|c|}
\hline & All patients & Non-Induction failure & Induction Failure & $\mathbf{P}$ \\
\hline Patients* & $109(100)$ & $66(61)$ & $43(39)$ & - \\
\hline Male patients" & $56(51)$ & $31(47)$ & $24(62)$ & n. s. \\
\hline Patient age (years) $)^{\dagger}$ & $58(18-75)$ & $57(18-75)$ & $60(20-75)$ & n. s. \\
\hline Sorror index (0-6) & $1.2(0-6)$ & $0.95(0-6)$ & $1.55(0-6)$ & 0.021 \\
\hline $\operatorname{ECOG}(0-3)$ & 0.76 & $0.61(0-3)$ & $0.81(0-3)$ & n. s. \\
\hline Positive CMV serology & $52(48)$ & $34(52)$ & $18(42)$ & n. s. \\
\hline LDH in IU/L(range) & $560(83-5820)$ & $646(132-5820)$ & $421(83-1693)$ & n. s. \\
\hline $\begin{array}{l}\text { Disease etiology }{ }^{*} \\
\text { de-novo } \\
\text { other }\end{array}$ & $\begin{array}{r}87(80) \\
13(20)\end{array}$ & $\begin{array}{l}56(85) \\
10(15)\end{array}$ & $\begin{array}{l}31(72) \\
15(28)\end{array}$ & n. s. \\
\hline $\begin{array}{l}\text { Disease genetic group } * \sharp \\
\text { Favourable (1) } \\
\text { Intermediate (2) } \\
\text { adverse (3) }\end{array}$ & $\begin{array}{l}15(14) \\
56(51) \\
38(35)\end{array}$ & $\begin{array}{l}13(20) \\
36(55) \\
17(26)\end{array}$ & $\begin{array}{c}2(5) \\
20(47) \\
21(49)\end{array}$ & $1 / 2$ vs 3 \\
\hline Blasts & $38(20-90)$ & $32.9(20-90)$ & $45.9(20-90)$ & 0.010 \\
\hline Platelets + & $104.1(8-1437)$ & $100.6(8-1437)$ & $109.4(16-479)$ & n. s. \\
\hline WBC+ & $28.7(0.1-302)$ & $29.2(0.1-302)$ & $27.9(1.2-244)$ & n. $s$. \\
\hline Biclonal leukemia detected by flow cytometer & $11(10)$ & $4(6)$ & $7(16)$ & n. s. \\
\hline Extramedullar AML & $5(5)$ & $3(5)$ & $2(5)$ & n. s. \\
\hline $\begin{array}{l}\text { Salvage Therapy } \\
\text { IDA-FLAG } \\
\text { Direct allo transplant } \\
\text { BSC }\end{array}$ & $\begin{array}{c}38 \\
30 \\
8 \\
5\end{array}$ & $\begin{array}{l}- \\
- \\
- \\
-\end{array}$ & $\begin{array}{c}38 \\
30 \\
8 \\
5\end{array}$ & n. d. \\
\hline
\end{tabular}

"number of patients $(\%),{ }^{\dagger}$ median (range), + average (range)

"favorable: $\mathrm{t}(8 ; 12)$ or $R U N X 1-R U N X 1 T 1$; inv(16)(p13q22); $\mathrm{t}(16 ; 16)(\mathrm{p} 13 \mathrm{q} 22)$ or $C B F B-M Y H 11 ; \mathrm{t}(15 ; 17)$; normal karyotype and mutated NPMI without FLT3-ITD or mutated CEBPA ${ }^{10}$ intermediate: Cytogenetic or molecular genetic abnormalities not classified as favorable or adverse ${ }^{10}$

adverse: inv(3)(q21q26.2) or t(3;3)(q21;q26.2) or RPN1-EVI1; $\mathrm{t}(6 ; 9)(\mathrm{p} 23 ; \mathrm{q} 34)$ or $D E K-N U P 214 ; \mathrm{t}(\mathrm{v} ; 11)(\mathrm{v} ; \mathrm{q} 23)$ or $M L L$ rearranged; -5 or del(5q); -7 ; abnl(17p); complex karyotype ${ }^{10}$ Abbreviations: CMV indicates human cytomegalovirus;WBC, white blood count, IDA-FLAG, idarubicin and fludarabine,BSC, best supportive care, n.s., not significant ( $\mathrm{p} \geq 0.05$ ), n.d.; not done. 
with previously published institutional standards [8,10,11]. The myeloablative preparative regimen consisted of 5 daily fractions of 30 $\mathrm{mg} / \mathrm{m}^{2}$ intravenous fludarabine (total dose $150 \mathrm{mg} / \mathrm{m}^{2}$ of body surface area [BSA]) with either 2 to 4 consecutive daily doses of $3,2 \mathrm{mg} / \mathrm{kg}$ of body weight of busulfan intravenously (total dose 6,4 to $12,8 \mathrm{mg} / \mathrm{kg}$ of body weight) or three consecutive daily intravenous doses of $10 \mathrm{~g} / \mathrm{m}^{2}$ treosulfan (total dose $30 \mathrm{~g} / \mathrm{m}^{2}$ of BSA). Patients with unrelated donor received additionally anti-thymoglobulin $10 \mathrm{mg} / \mathrm{kg} / \mathrm{BW}$ for three days.

The pharmacologic prophylaxis of acute GvHD consisted of bloodlevel adjusted continuous intravenous ciclosporin (CSP) combined with short-course methotrexate or mycophenolate-mofetil [10].

\section{Study endpoints and statistical analysis}

Mann-Whitney U-test and one-sided Fisher-exact test was used to test differences of continuous variables. For the comparison of overall survival (OS), relapse incidence (RI), and leukemia-free survival (LFS), the probabilities of events over time were calculated by the productlimit method and heterogeneity of time-to-event distribution function was compared by the log-rank [12]. For the comparison of cumulative incidence (C.I.) rates between patient subsets, the time-to-events were compared by proportional hazards general linear model (PHGLM) analysis of the cause-specific hazard functions using the two-sided Wald test. In addition, multivariate PHGLM analysis of the endpoints of LFS and OS was performed $[12,13]$. In all multivariate PHGLM analyses of these endpoints the following dichotomous covariates were included as time-dependent with the time interval from time of diagnosis until the first occurrence of the respective event or censoring in model building: CMV IgG serostatus (positive versus negative), LDH-value (norm or upper norm), platelet counts (over $40.000 / \mathrm{nL}$ or below), blast count in bone marrow over $40 \%$ or below, Sorror comorbidity score (range $0-6)$ over 1 or below, age ( $<70$ years or above) [14], cytogenetic risk factors according to the ELN classification (favourable, intermediate, or high risk), occurrence of biclonal AML detected by flow cytometry and extramedullary manifestation of AML. All PHGLM analyses were performed using stepwise forward and backward selection procedures and only those covariates with a significance level below $5 \%$ were allowed to enter the model building procedure. The hazard ratio (HR) and its 95\% confidence interval (95\%-CI) were derived for each significant covariate included in the final PHGLM models. Statistical analysis and presentation was performed using SPSS-20 Software ${ }^{\mathrm{mm}}$ procedures.

\section{Results}

\section{Incidence of IF and salvage therapy for IF after DA}

In 43 of 109 patients (39\%), who received one or two courses of 7+3 DA an IF had been documented. 38 of 43 patients received a salvage therapy with either a combination of fludarabine and idarubicin (FLAG-IDA, n=30) and thereafter alloSCT or proceeded directly to alloSCT $(n=8)$. Notably 5 of 43 patients with IF received no further antileukemic therapy for AML, but only best supportive care. Interestingly, the occurrence of IF had no influence on overall survival outcome when subsequently an alloSCT was performed.

\section{Risk factors for IF}

Age over 69 years $(\mathrm{p}=0.020)$, Sorror index $>1 \quad(\mathrm{p}=0.019)$, unfavourable cytogenetics $(\mathrm{p}=0.014)$ and blast count $>40 \%$ in bone marrow $(\mathrm{P}=0.004)$ had influence on the occurrence of IF, whereas a positive CMV IgG sero-status, ECOG 0-1 versus 2-4, low platelet count $<40.000 / \mathrm{nL}$, biclonary AML detected by flow cytometry, LDH over norm, detection of extramedullary AML had no influence statistically on the occurrence of IF after DA as shown in Table 2.

\section{IF and relapse}

The median time of follow-up for the whole cohort was 18 months (1-65 months). Relapse was detected in 21 of 109 patients. Occurrence of IF had no influence on relapse rate in patients with AML when subsequently an allogeneic transplant was performed. The probability for 2-year relapse incidence was $40 \%+11.4 \%$ without allogeneic transplant versus $17.8 \%+5.7 \%, \mathrm{p}=0.009$ with transplant as shown in Figure 1. Blast count $>40 \%$ at time point of diagnosis had a higher probability of relapse (2-year cumulative incidence of relapse was for blasts $>40 \%(37.5 \%+9.2 \%$ versus only $15.1 \%+6.3 \%$ for blasts $<40 \%$, $\mathrm{p}=0.0364)$. Further factors like adverse cytogenetic, age over 70 years, ECOG status over 2, Sorror index of more than 1 did not achieved significance statistically. Other factors as extramedullary AML, biclonal AML detected by flow cytometry and positive CMV IgG sero-status also had no effect statistically on the occurrence of relapse. In multivariate analysis only the performance of an allogeneic transplant (HR 0.29, [95\%CI: $0.12-0.72$ ], $\mathrm{p}=0.008$ ) and blast count $>40 \%$ in bone marrow (HR 2.91, [95\%CI: $1.13-7.48$ ], $\mathrm{p}=0.027$ ) were identified as risk factors for relapse.

Table 2. Risk factors for induction failure

\begin{tabular}{|c|c|c|c|c|c|}
\hline & All patients & Non-Induction failure & Induction Failure & $\mathbf{p}$ & Odd-Ratio \\
\hline Patients* & $109(100)$ & $66(61)$ & $43(39)$ & - & \\
\hline $\begin{array}{l}\text { Patient' age (years) }{ }^{*} \\
\text { Between } 18 \text { and } 69 \\
\text { Older than } 69\end{array}$ & $\begin{array}{l}84(77) \\
25(23)\end{array}$ & $\begin{array}{l}55(83.3) \\
11(16,7)\end{array}$ & $\begin{array}{l}29(67) \\
14(33)\end{array}$ & 0.020 & 2.5 \\
\hline Sorror index >1 (\%) & $13(19.7)$ & 13 & 18 & 0.019 & 2.72 \\
\hline $\begin{array}{l}\text { Disease genetic group*\$ } \\
\text { Favourable (1) } \\
\text { Intermediate (2) } \\
\text { adverse (3) }\end{array}$ & $\begin{array}{l}15(14) \\
56(51) \\
38(35)\end{array}$ & $\begin{array}{l}13(20) \\
36(55) \\
17(26)\end{array}$ & $\begin{array}{c}2(5) \\
20(47) \\
21(49)\end{array}$ & $\begin{array}{c}1 / 2 \text { vs } 3 \\
0.014\end{array}$ & 3.21 \\
\hline Blasts* $>40 \%$ & $35(32)$ & $15(23)$ & $20(47)$ & 0.004 & 3.64 \\
\hline
\end{tabular}

"number of patients $(\%),{ }^{+}$median (range), \# average (range)

"favorable: $\mathrm{t}(8 ; 12)$ or RUNX1-RUNXIT1; inv(16)(p13q22); $\mathrm{t}(16 ; 16)(\mathrm{p} 13 \mathrm{q} 22)$ or $C B F B-M Y H 11 ; \mathrm{t}(15 ; 17)$; normal karyotype and mutated $N P M 1$ without FLT3-ITD or mutated CEBPA ${ }^{10}$ intermediate: Cytogenetic or molecular genetic abnormalities not classified as favorable or adverse ${ }^{10}$

adverse: inv(3)(q21q26.2) or t(3;3)(q21;q26.2) or RPN1-EVI1; $\mathrm{t}(6 ; 9)(\mathrm{p} 23 ; \mathrm{q} 34)$ or $D E K-N U P 214 ; \mathrm{t}(\mathrm{v} ; 11)(\mathrm{v} ; \mathrm{q} 23)$ or $M L L$ rearranged; -5 or del(5q); 7 ; abnl(17p); complex karyotype ${ }^{10}$ Abbreviations: HCMV indicates human cytomegalovirus; TBI, total body irradiation; sMTX, short-course methotrexate; CsA,cyclosporine A; MMF, mycophenolate mofetil; and n. s., not significant $(\mathrm{p} \geq 0.01)$. 


\section{IF and leukemia-free survival (LFS) and OS}

Interestingly, the occurrence of IF had no influence on OS or LFS outcome when an allogeneic transplant was performed subsequently (2-year OS $86.9 \%+7.6 \%$ versus $19.4 \%+16.8 \%, \mathrm{p}=0.0001$ ) (Figures 2 and 3). In a univariate analysis risk factors for LFS were age over 70 years (2-year-LFS $65.5 \%+6 \%$ versus $35.6 \%+12 \%, p=0.002)$, ECOG $0-2$ versus $3-4$ (2-year LFS $60.4 \%+5.7 \%$ versus $54 \%+15 \%, p=0.023)$, bone marrow blast count under $40 \%$ at diagnosis (2-year-LFS $69.7 \%+$ $7 \%$ versus $42.8 \%+8.4 \%, \mathrm{p}=0.028)$, and the performance of allogeneic transplant $(2$-year LFS $68.3 \%+6.5 \%$ versus $41.5 \%+9.4 \%, \mathrm{p}=0.001)$ as shown in Figures 4-7. No statistical influence on LFS was found for Sorror comorbidity index of more than 1 and biclonal AML as detected by flow cytometry.

Risk factors for OS were age over 70 years (2-year OS $71.4 \%+$ $5.8 \%$ versus $57.2 \%+12.3 \%, \mathrm{p}=0.046)$, biclonal AML detected by flow cytometry (2-year OS $70.7 \%+5 . \%$ versus $47.7 \%+17.6 \%, \mathrm{p}=0.033$ ), bone marrow blast count more than $40 \%$ (2-year OS $79.1 \%+6.1 \%$ versus $52.1 \%+8.9 \%, \mathrm{p}=0.033)$, and the performance of allogeneic transplant (2-year OS $76.2 \%+6.2 \%$ versus $63.1 \%+8.4 \%, \mathrm{p}=0.003$ ).
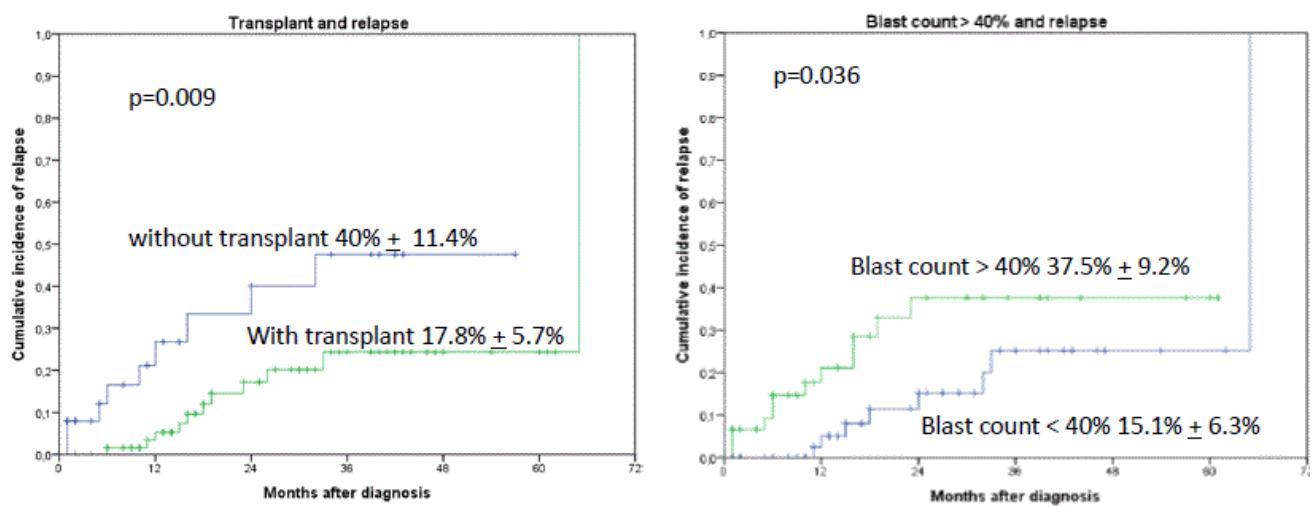

Figures 1A and B. Cumulative incidence of relapse (CIR) stratified by occurrence of IF and allogeneic transplant. The 2-year CIR was $40 \%+11.4 \%$ without allogeneic transplant versus $17.8 \% \pm 5.7 \%$, $\mathrm{p}=0.009$ with transplant. B. Blast count $>40 \%$ at time point of diagnosis had a higher probability of relapse than blast count $<40 \%(2-$ year CIR was for blasts $>40 \% 37.5 \%$ $\pm 9.2 \%$ versus only $15.1 \% \pm 6.3 \%$ for blasts $<40 \%, \mathrm{p}=0.036)$
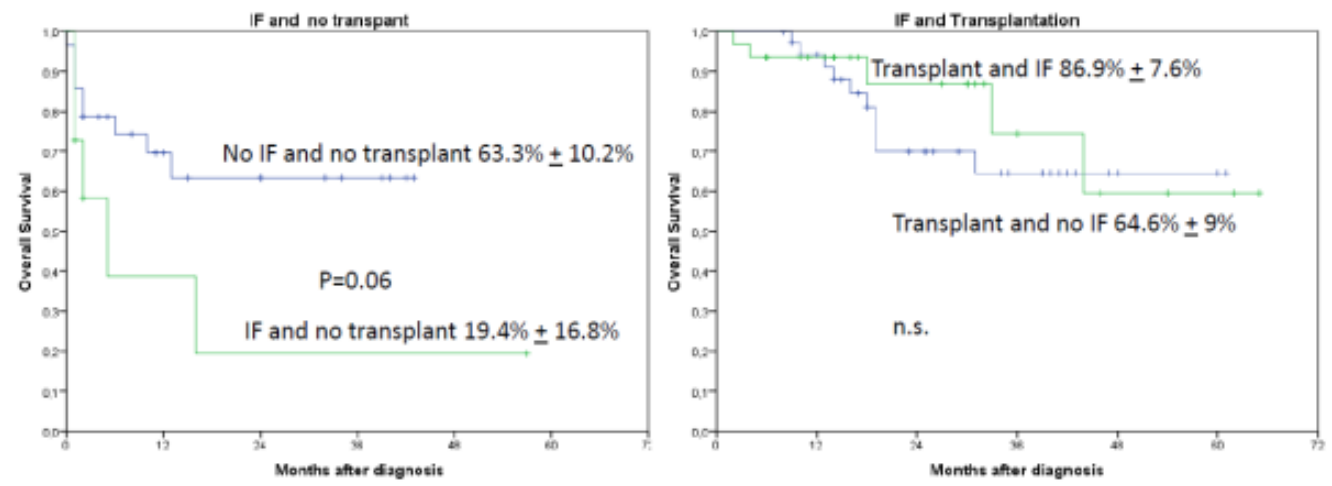

Figures 2A and B. A shows the influence of IF and no transplant on OS. The 2 -year OS was for patients without transplant and no induction failure $63.3 \%+10.2 \%$ versus $19.4 \%+16.8 \%$ $\mathrm{P}=0.06$ for patients with induction failure and transplant. Patients with IF and transplant compared to patients with transplant and no IF had a 2 -year OS of $86.9 \%+7.6 \%$ versus $64.6 \%$ $+9 \%$, respectively. Patients with IF and transplant compared to patients with IF and without transplant (A and B) had a poor outcome with a 2 -year OS of $86.9 \%+7.6 \%$ versus $19.4 \%+$ $16.8 \%, \mathrm{p}=0.0001)$
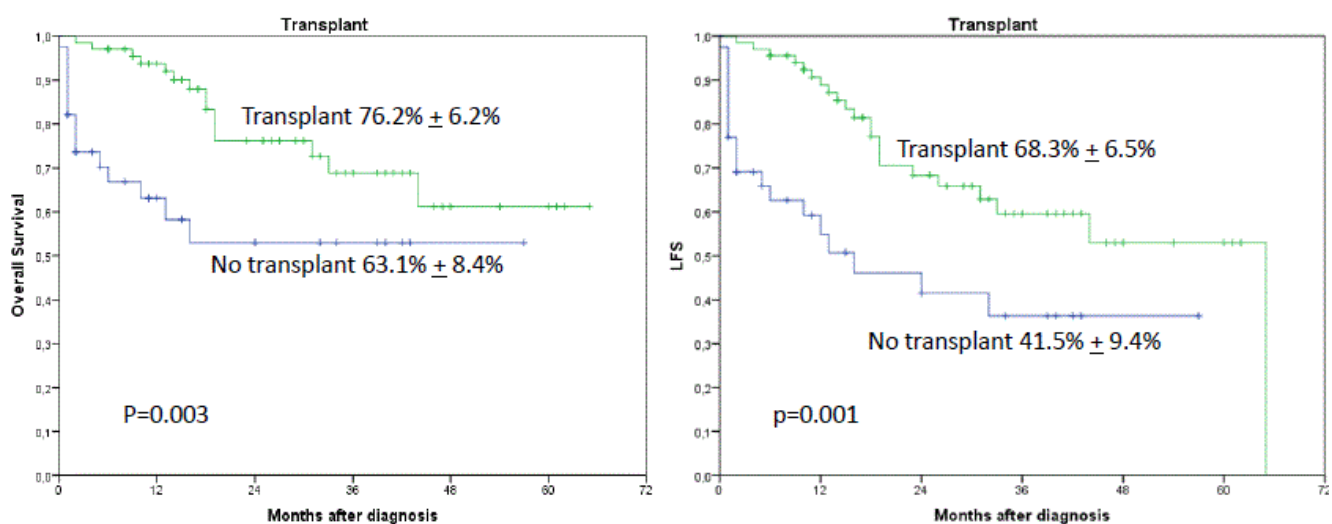

Figures 3A and B. A shows the influence of transplant on OS. The 2-year OS was $76.2 \%+6.2 \%$ when a transplant was performed regardless of occurrence of induction failure versus $63.1 \%$ $\pm 8.4 \%, p=0.003$, when no transplant was performed. B shows the influence on LFS when transplant was performed $(68.3 \% \pm 6.5 \%$ versus $41.5 \% \pm 9.4 \%$, $\mathrm{p}=0.001)$ 

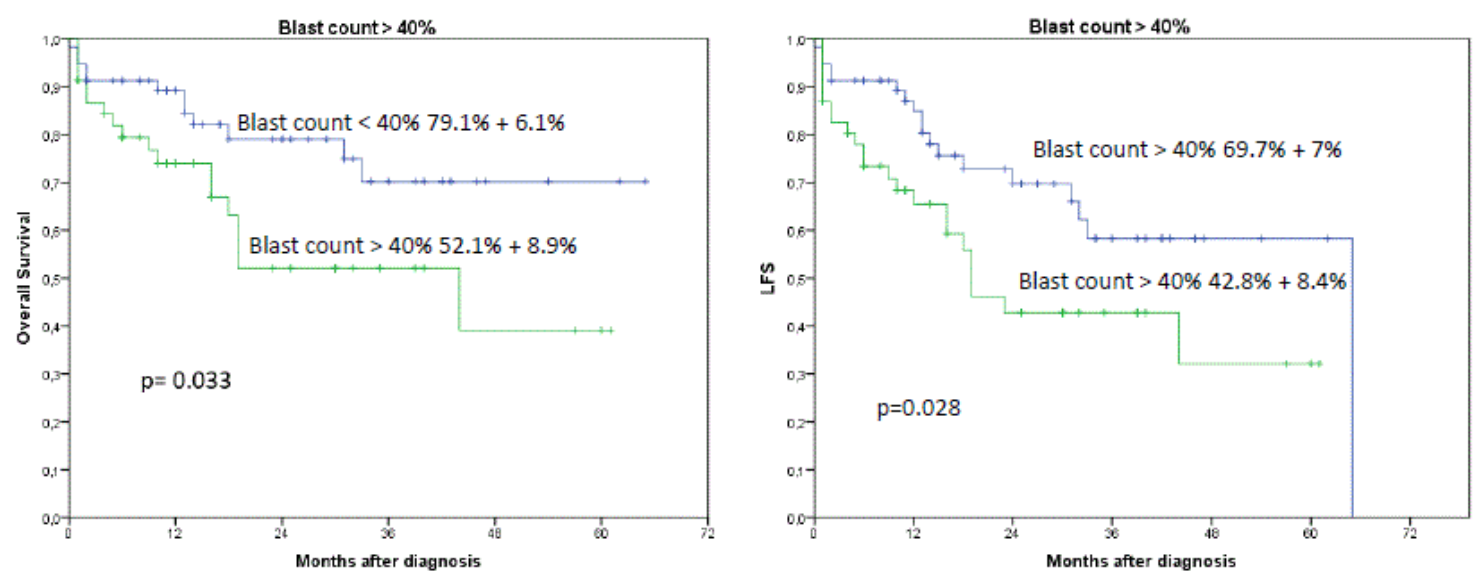

Figures $4 \mathrm{~A}$ and B. A shows the influence of blast count $>40 \%$ in bone marrow on OS and LFS regardless of occurrence of induction failure. The 2 -year OS was $79.1 \%+6.1 \%$ for blast count $<40 \%$ versus $52.1 \% \pm 8.9 \%$ for blast count $>40 \%, \mathrm{p}=0.033$. B shows the influence on LFS for blast count $>40 \%$ in bone marrow (2-year LFS was $69.7 \% \pm 7 \%$ for blast count $<40 \%$ versus $42.8 \% \pm 8.4 \%$ for blast count $>40 \%, \mathrm{p}=0.028$ )
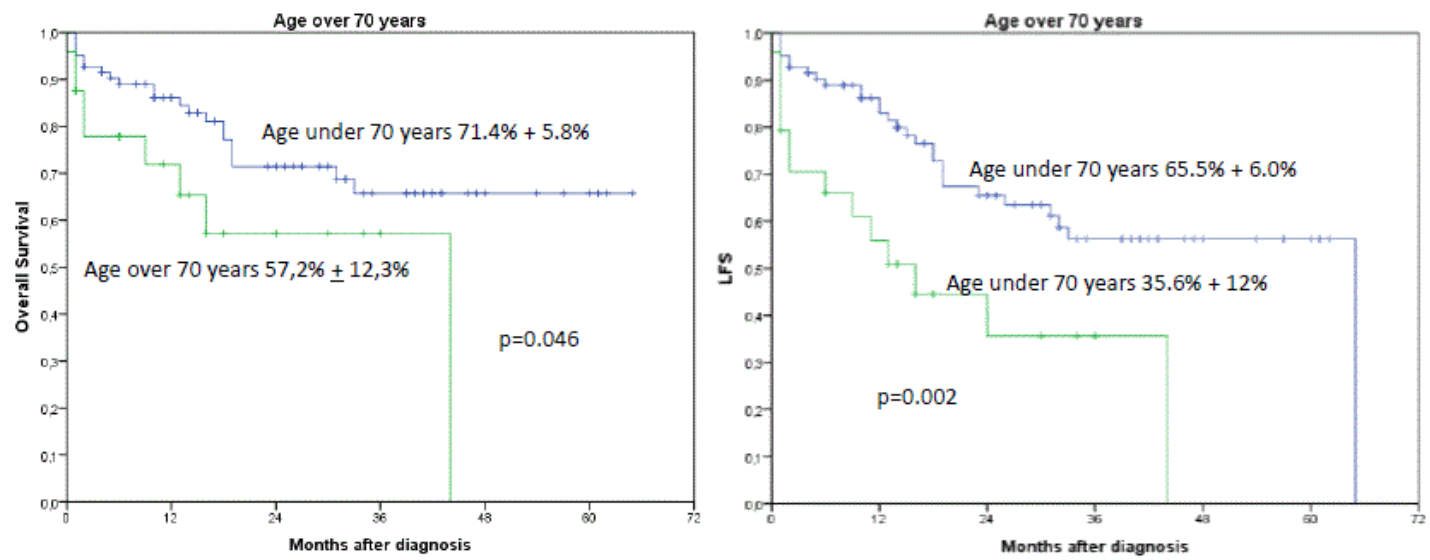

Figures 5A and B. B shows the influence of age $>70$ years on OS and LFS regardless of occurrence of induction failure. The 2-year OS was $71.4 \% \pm 5.8 \%$ for patients under the age of 70 years versus $57.2 \% \pm 12.3 \%$ for patients older than 70 years, $\mathrm{p}=0.046$. B shows the influence of age over 70 years on LFS. (2-year LFS $65.5 \% \pm 6.0 \%$ versus $35.6 \pm 12 \%$ for patients older than 70 years, $\mathrm{p}=0.002$ )
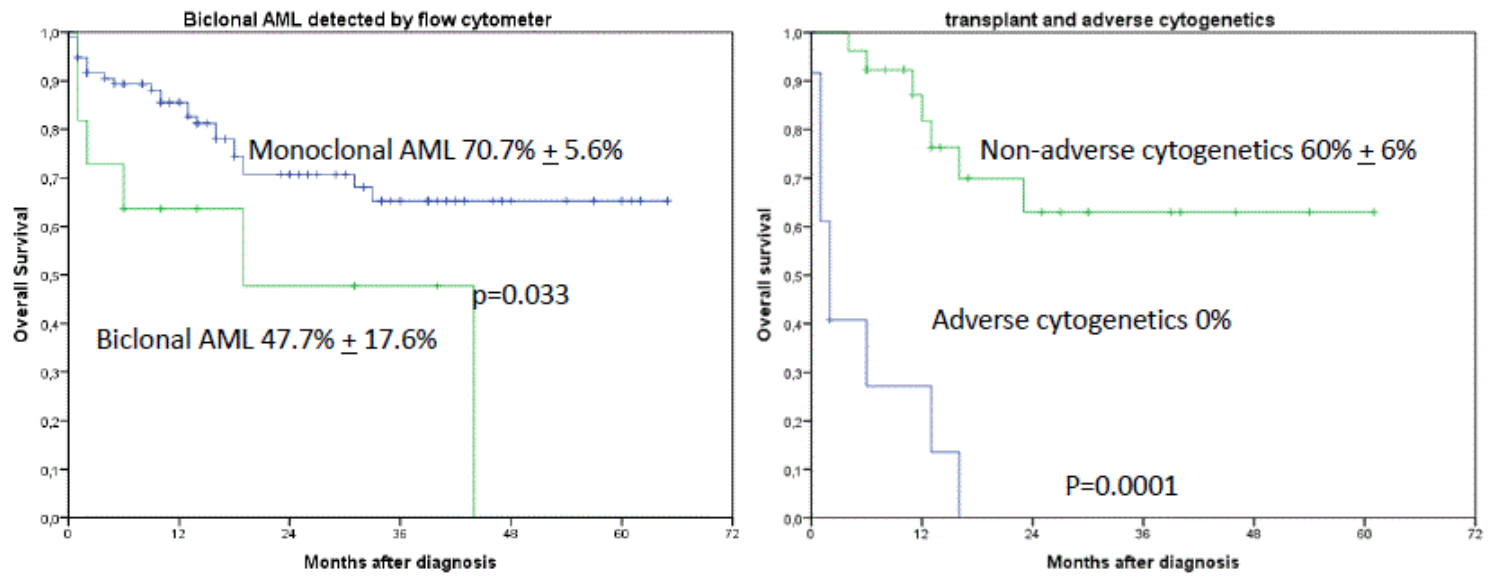

Figures 6A and B. A shows the influence of biclonal AML detected by flow cytometry on OS regardless of occurrence of induction failure. The 2 -year OS was $70.7 \% \pm 5.6 \%$ for monoclonal AML versus $47.7 \% \pm 17.6 \%$ for biclonal AML, $\mathrm{p}=0.033$. B shows the influence of transplant and adverse cytogenetics on OS $(2$-year OS was $60 \% \pm 6 \%$ when allogeneic transplant was performed versus $0 \%$ when no transplant was performed, $\mathrm{p}=0.001$ ) 


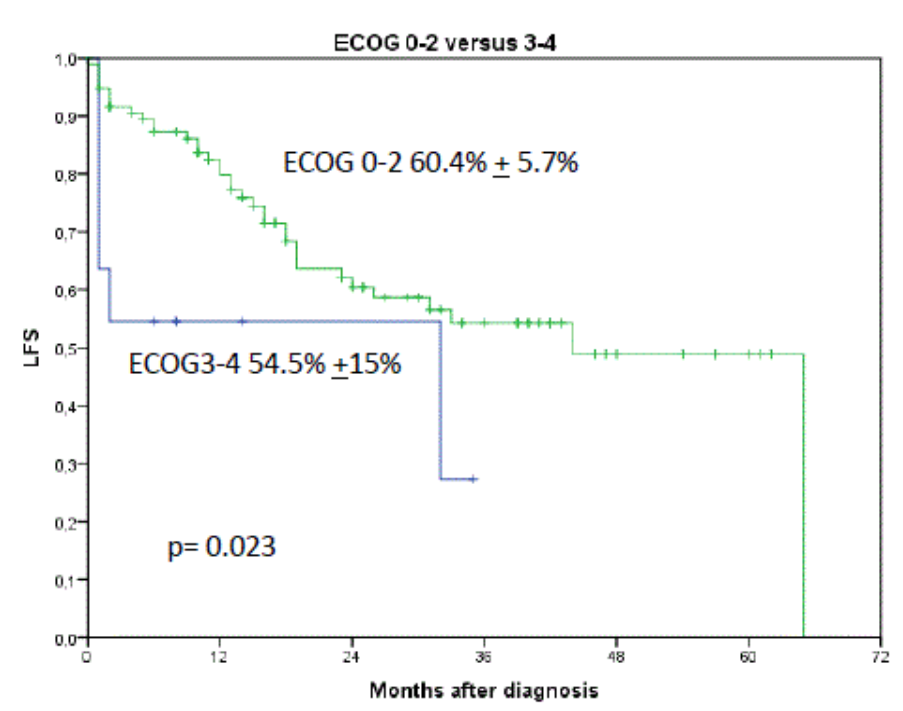

Figure 7. Shows the influence of ECOG performance status on LFS. 2-year LFS for patients with ECOG $0-2$ was $60.4 \% \pm 5.7 \%$ versus $54.5 \% \pm 15 \%$ for ECOG $3-4, \mathrm{p}=0.023$

Adverse cytogenetics were associated with poor outcome when no allogeneic transplant was performed (2-year OS $0 \%$ versus $62.9 \%+$ $11.6 \%, \mathrm{p}=0.0001$, Figure 6).

All other factors achieved no significance statistically.

\section{Multivariate analyses for OS and LFS}

In the multivariate analysis of risk factors for LFS only transplantation (HR 0.36, [95\% CI 0.19 - 0.67], $\mathrm{p}=0.001$, age over 70 years (HR 2.41, [95\% CI $1.26-4.67], \mathrm{p}=0.009$ ) and blast count $>40 \%$ in bone marrow (HR 2.08, [95\% CI 1.09-3.89], $\mathrm{p}=0.025$ ) were identified. For OS only transplantation (HR 0.37; [95\% CI 0.19-0.76], $\mathrm{p}=0.007$ ) and blast count $>40 \%$ in bone marrow (HR 2.24, [95\% CI $1.07-4.68$ ], $\mathrm{p}=0.032$ ) were identified as risk factors, which influenced the outcome. IF, cytogenetics, biclonal AML detected by flow cytometry, Sorror comorbidity index, ECOG (0-2) performance status had no influence on either LFS or OS.

\section{Discussion}

Despite the new era with targeted therapies as FLT-3 inhibitors e.g. midostaurin, quizartinib, or crenolanib and IDH1/2 inhibitors e.g. enasidenib and ivosidenib induction therapy with DA is still the backbone worldwide in the treatment for AML [15-17]. While a plethora of scientific publications for AML have substantiated an impact of older age, cytogenetics according to the ELN classification and allogeneic transplantation on the outcome of AML, only little information on risk factors for IF and its frequency after standard induction therapy with DA, is currently available $[1,7,9,18]$. Moreover, the frequency of the occurrence of IF in AML is often underestimated [3]. Additionally, increasingly older patients are treated in a curative intention for AML and proceed to alloSCT. These patients must however first surpass the steep slope of induction chemotherapy with DA, which can lead to a substantial increase in the rate of IF. It is therefore important to evaluate the real frequency of IF as done in this study $[18,19]$.

In this present study we report a high rate of IF with $39 \%$ after standard induction therapy with DA in patients who were consecutively treated for AML at our centre. Recently, Wattad and coworker evaluated the outcome of five prospectively performed studies with 3324 patients and reported an incidence of IF in 30.8\% (1025 of
3324 patients) after the use of a more intensive induction therapy with idarubicin, cytarabine and etoposide (ICE) compared to the standard induction therapy with DA. The higher rate of IF that is reported in our study might be caused by an higher median age of 59 years compared to 55 years [20]. The inclusion of a higher rate of patients over 60 years in our AML study is commonly accepted to be associated with a higher proportion of patients with unfavourable cytogenetics as mentioned above. Accordingly, our study cohort consists of a lower rate of patients with favourable cytogenetics according to the ELN classification with $14 \%$ compared to the above mentioned study by Wattad and co-workers with more than $25 \%$, which might explain the higher rate of IF in our study compared to that of Wattad and co-workers [20].

Beside the known risk factors age over 70-years and adverse cytogenetics according to the ELN-classification, we also identified Sorror comorbidity score of over 1, and blast count more than $40 \%$ in bone marrow as risk factors for the occurrence of IF. Notable, more than $40 \%$ blast count in bone marrow was the strongest factor. Further of interest was that the occurrence of IF had no influence on overall survival when subsequently an allogeneic transplantation was performed, whereas not to perform a subsequent transplantion was associated with poor outcome (2-year OS $86.9+7.6 \%$ for transplant versus $19.4 \%+16.8 \%$ for no transplant, $\mathrm{p}=0.0001$ ).

Generally, the prognosis of AML-patients with primary IF is reported to be extremely poor. Miyamara and co-workers reported in paediatric AML-patients with primary induction failure registered to the Japanese Paediatric Leukaemia/Lymphoma Study Group AML-05 study a 3-year OS rate of only $19.0 \%$. High leucocyte count, M7 morphology, and unfavourable genetic aberrations, such as FLT3-internal tandem duplication, NUP98-NSD1 and high MECOM or PRDM16 expression, were risk factors for IF in their study [21]. Better survival probabilities were reported, by Wattad and co-workers, in patients with IF responding to salvage therapy and receiving subsequently alloSCT as reported here in our study [20]. Our finding confirms a poor outcome of patients after IF without subsequent performance of an allogeneic transplantation. In our multivariate analysis, we found that allogeneic transplantation and blast count over $40 \%$ were the only independent risk factors for OS. In the present study the occurrence of adverse cytogenetics was a risk factor for OS too, when no transplant was performed as found by other studies.

Accompanying diseases and performance status certainly influence the outcome of AML as we and others have shown [18]. Here we presented data showing that Sorror comorbidity index of more than 1 is a risk factor for the occurrence of IF. Furthermore, that a restricted performance status of ECOG more than 2 has influence on the outcome for LFS.

In a previously performed study we had shown that there is evidence suggesting that cytomegalovirus (CMV) reactivation following allogeneic hematopoietic stem cell transplantation is associated with a lower relapse incidence in AML [22,23]. Therefore, we investigated here the association of the occurrence of a positive CMV IgG sero-status with the relapse risk of AML and to occurrence of IF, which we could not demonstrate here probably due to the low number of study patients.

Interestingly we found that the occurrence of biclonal AML detected by flow cytometry had influence on the LFS, showing that several clones of AML at time point of diagnosis is of importance in the outcome of patients. Hereby we restricted our analysis of biclonality of AML to analysis by flow cytometry in order to consider only a second AML population with a minimum of $2 \%$ of all blasts. Their influence was not detectable on LFS in the multivariate analysis. 
In conclusion, this present study provides data characterising risk factors for the occurrence of IF and shows that a subsequent alloSCT as an independent factor equalizes this obstacle. The study highlights the urgent need for more effective induction approaches in elderly AML patients, especially to facilitate an increase in elderly patients, who proceed to alloSCT.

A simple increase in anthracycline dose of induction therapy for patients older than 60 years to achieve higher rates of complete remission as suggested by Lowenberg and co-workers is not often applicable due to the comorbidities of older patients [18]. Indeed, patients over 70 years represent the majority of patients with AML. The use of liposomal daunorubicin and cytarabine or combinations of targeted therapies with Venetoclax and Azacitidine might be an option for older patients to reduce the high frequency of IF as reported in this study here [24-26].

\section{Acknowledgements}

The authors are indebted to the technicians of the Department of Hematology at the AK St.Georg for their excellent technical long-term support in the performance of flow cytometry analysis. They are particulary grateful for the provision of cytogenetic and molecular disease-specific data provided by the co-workers at the Universitätsmedizin Göttingen Laboratory (MLL), Göttingen, Germany.

\section{Authorship contributions}

\section{Conception and design: Ahmet H. Elmaagacli}

Administrative support: Ahmet H. Elmaagacli

Provision of study materials and patients: Ahmet H. Elmaagacli

Collection and assembly of data: Christian Jehn, Marc Pannenbeckers, Anna Klapproth, Farouk Dahmash, Hans Salwender, Anju Singh, Yana Shikova, Jara Pascale Schlichting, Silke Meyer, Cornelius Niggemann, Mathias Vierbuchen and Ahmet H. Elmaagacli,

Data analysis and interpretation: Ahmet H. Elmaagacli, Marc Pannenbeckers, Anna Klapproth, Christian Jehn

\section{Manuscript writing: Ahmet H. Elmaagacli, Christian Jehn}

Final approval of manuscript: Christian Jehn, Marc Pannenbeckers, Anna Klapproth, Farouk Dahmash, Hans Salwender, Anju Singh, Yana Shikova, Jara Pascale Schlichting, Silke Meyer, Cornelius Niggemann, Mathias Vierbuchen and Ahmet H. Elmaagacli.

\section{Conflicts of Interest Disclosures}

The authors MP, AK, FD, HS, AS, YS, JPS, SM, CN, MV declare no potential conflicts of interest. AHE and CFJ received a speaker honorarium from JAZZ and Daiichi-Sankyo.

\section{References}

1. Schott DH, Collins RN, Bretscher A (2002) Secretory vesicle transport velocity in living cells depends on the myosin V lever arm length. J Cell Biol 156: 35-39.

2. Crowther D, Powles RL, Bateman CJ, Beard ME, Gauci CL, et al. (1973) Management of adult acute myelogenous leukaemia. Br Med J 5846: 131-137.

3. Fröhling S, Schlenk RF, Kayser S, Morhardt M, Benner A, et al. (2006) Cytogenetics and age are major determinants of outcome in intensively treated acute myeloid leukemia patients older than 60 years: results from AMLSG trial AML HD98-B. Blood 108: $3280-3288$

4. Megías-Vericat JE, Martínez-Cuadrón D, Sanz MA, Montesinos P (2018) Salvage regimens using conventional chemotherapy agents for relapsed/refractory adult AML patients: A systematic literature review. Annals of Hematology 97: 1115-1153.
5. Holtick U, Shimabukuro-Vornhagen A, Chakupurakal G, Theurich S, Leitzke S, et al. (2016) FLAMSA reduced-intensity conditioning is equally effective in AML patients with primary induction failure as well as in first or second complete remission. Eur $J$ Haematol 96: 475-482. [Crossref]

6. Kim DY, Lee JH, Sym SJ, Yun SC, Lee JH, et al. (2011) A prediction model for complete remission upon reinduction for patients with acute myeloid leukemia after failure of anthracycline and cytarabine standard chemotherapy. Ann Hematol 90: 12831291.

7. Lazzarotto D, Candoni A, Nadali G, Pavan L, Lessi F, et al. (2016) Multicentre survey to explore current survival of patients with acute myeloid leukaemia who failed induction chemotherapy. Eur J Haematol 96: 586-92.

8. Juliusson G, Antunovic P, Derolf A, Lehmann S, Mollgard L, et al. (2009) Age and acute myeloid leukemia: real world data on decision to treat and outcomes from the Swedish Acute Leukemia Registry. Blood. 113: 4179-87.

9. Ottinger HD, Ferencik S, Beelen DW, Lindemann M, Peceny R, et al. (2003) Hematopoietic stem cell transplantation: contrasting the outcome of transplantations from HLA-identical siblings, partially HLA-mismatched related donors, and HLAmatched unrelated donors. Blood 102: 1131-1137.

10. Döhner H, Estey EH, Amadori S, Appelbaum FR (2010) Diagnosis and management of acute myeloid leukemia in adults: Recommendations from an international expert panel, on behalf of the European LeukemiaNet. Blood 115: 453-474.

11. Gratwohl A (2008) Principles of conditioning. In: Apperley J, Carreras E, Gluckman E, Gratwohl A, Masszi T eds. The ESH-EBMT Handbook on Haematopoietic Stem Cell Transplantation 129-144.

12. Elmaagacli AH, Peceny R, Steckel N (2003) Outcome of transplantation of highly purified peripheral blood CD34+ cells with T-cell add-back compared with unmanipulated bone marrow or peripheral blood stem cells from HLA-identical sibling donors in patients with first chronic phase chronic myeloid leukemia. Blood 101: 446453.

13. Kaplan EL, Meier P (1958) Nonparametric estimation from incomplete observations. $J$ Am Stat Assoc 53: 457-481.

14. Cox DR (1972) Regression models and life-tables (with discussion). J Royal Stat Soc B34: 187-220.

15. Sorror ML, Maris MB, Storb R, Baron F, Sandmaier BM, et al. (2005) Hematopoietic cell transplantation (HCT)-specific comorbidity index: a new tool for risk assessment before allogeneic HCT. Blood 106: 2912-19.

16. Stone RM, Mandrekar SJ, Sanford BL, Laumann K, Geyer S, et al. (2017) Midostaurin plus Chemotherapy for Acute Myeloid Leukemia with a FLT3 Mutation. $N$ Engl J Med 377: 454-464.

17. Cortes J, Perl AE, Döhner H, Kantarjian H, Martinelli G, et al. (2018) Quizartinib, an FLT3 inhibitor, as monotherapy in patients with relapsed or refractory acute myeloid leukaemia: an open-label, multicentre, single-arm, phase 2 trial. Lancet Oncol 19: 889903. [Crossref]

18. Pollyea DA, Tallman MS, de Botton S, Kantarjian HM, Collins R, et al. (2019) Enasidenib, an inhibitor of mutant IDH2 proteins, induces durable remissions in older patients with newly diagnosed acute myeloid leukemia. Leukemia 33: 2575-2584.

19. Löwenberg B, Ossenkoppele GJ, van Putten W, Schouten HC, Graux C, et al. (2009) High-dose daunorubicin in older patients with acute myeloid leukemia. $N$ Engl J Med 361: $1235-48$.

20. Bell JA, Galaznik A, Farrelly E, Blazer M, Murty S, et al. (2009) A retrospective study evaluating treatment patterns and survival outcomes in elderly patients with acute myeloid leukemia treated in the United States with either $7+3$ or a hypomethylating agent. Leukemia Research 78: 45-51.

21. Wattad M, Weber D, Döhner K, Krauter J, Gaidzik VI, et al. (2017) Impact of salvage regimens on response and overall survival in acute myeloid leukemia with induction failure. Leukemia 31: 1306-1313.

22. Miyamura T, Moritake H, Nakayama H, Tanaka S, Tomizawa D, et al. (2019) Clinical and biological features of paediatric acute myeloid leukaemia (AML) with primary induction failure in the Japanese Paediatric Leukaemia/Lymphoma Study Group AML05 study. Br J Haematol 185: 284-288.

23. Elmaagacli AH, Steckel NK, Koldehoff M, Hegerfeldt Y, Trenschel R, et al. (2011) Early human cytomegalovirus replication after transplantation is associated with a decreased relapse risk: evidence for a putative virus-versus-leukemia effect in acute myeloid leukemia patients. Blood 118: 1402-12.

24. Elmaagacli AH, Koldehoff M (2016) Cytomegalovirus replication reduces the relapse incidence in patients with acute myeloid leukemia. Blood 128: 456-9. 
Jehn CF (2019) Risk factors for induction failure of standard chemotherapy with anthracycline and cytarabine in Acute Myeloid Leukemia Patients

25. Lancet JE, Uy GL, Cortes JE, Newell LF, Lin TL, et al. (2018) CPX-351 (cytarabine and daunorubicin) liposome for injection versus conventional cytarabine plus daunorubicin in older patients with newly diagnosed secondary acute myeloid leukemia. J Clin Oncol 36: 2684-2692.
26. DiNardo CD, Pratz K, Pullarkat V, Jonas BA, Arellano M, et al. (2019) Venetoclax combined with decitabine or azacitidine in treatment-naive, elderly patients with acute myeloid leukemia. Blood 133: 7-17. [Crossref]

Copyright: (C2019 Jehn CF. This is an open-access article distributed under the terms of the Creative Commons Attribution License, which permits unrestricted use, distribution, and reproduction in any medium, provided the original author and source are credited. 